\title{
Outcomes of Allogeneic Stem Cell Transplant (allo-SCT) Recipients in the Era of Newer Antifungal Agents
}

\author{
Nishi Shah², 1 , Ajay K. Nooka ${ }^{1}$, Sagar Lonial ${ }^{1}$, Hannah J. Khoury ${ }^{1}$, Edmund Waller ${ }^{1}$ and \\ Amelia Langston ${ }^{1}$
}

${ }^{1}$ Winship Cancer Institute, Emory University, Atlanta, GA, USA; ${ }^{2}$ Rollins School of Public Health, Emory university, Atlanta, GA, USA

\section{Introduction}

Fungal infections (FI) are a leading cause of morbidity and mortality among patients undergoing allo-SCT. The newer anti-fungal agents, the echinocandins and extended spectrum azoles, have offered alternatives to Amphotericin B and fluconazole. Data from large patient samples evaluating the magnitude of benefit with the newer anti-fungal agents are lacking. We analyzed the Nationwide Inpt Sample (NIS) database from Healthcare Cost and Utilization project to evaluate the trends in the incidence of FI and to evaluate the potential impact of newer anti-fungal agents on in-hospital mortality (IHM) among allo-SCT recipients.

\section{Methods}

We used the NIS database to obtain the inpatient data of adults admitted for primary procedures of allo-SCT between $01 / 2000$ until $12 / 2010$. We have evaluated trends of various FI in this patient population. We performed multivariate logistic regression analyses to evaluate the risk factors for IHM in allo-SCT pts. The occurrence of FI, the era of antifungal therapy (2000-2001 vs. 2002-2010), gender, payer status, and lengthy hospital stay ( $>$ median) were the variables used for the analyses. Lastly, we performed separate multivariate logistic regression analyses for 2000-2001 and 2002-2010 data to evaluate the difference in association of FI with IHM in allo-SCT pts.

\section{Results}

The incidence of invasive candidial infections, zygomycosis and other fungal infections (include histoplasmosis, coccidioidomycosis, allescheriosis, blastomycosis, paracoccidiodomycosis, lobomycosis, rhinosporidiosis, sporotrichosis, chromoblastomycosis, cryptococcosis, mycotic mycetomas, deep dermatophytosis) among allo-SCT patients has remained relatively stable over time, while aspergillosis has decreased slightly(fig 1). The occurrence of FI, medicare as payer status, allo-SCT done prior to the era of newer antifungal agents (2000-2001) and >median length of in-hospital stay were associated with higher IHM among allo-SCT patients (Odds ratio (OR) 4.331, p-value $<0.0001$, OR 1.359 , p-value $=0.0145$, OR 1.830 , p-value $=$ $<0.0001$, OR 3.017, p-value $<0.0001$ respectively) (Table 1). On separate analyses, we see that FI are more strongly associated with IHM in 2000-2001 (5.009, p-value <0.001) as compared to 2002-2010 (4.168, p-value $<0.001)$.

\section{Conclusions}

While the incidence of various FI among allo-SCT recipients has remained relatively stable over the last decade, the occurrence of FI, medicare as payer status and allo-SCT performed prior to the era of newer anti-fungal agents were both factors associated with higher IHM. Although there is a difference in ORs on separate analyses of the two eras, there is an overlap of confidence intervals (Table 1 ). This could possibly due to the small sample size. Further studies looking back in time prior to 2000 and looking further ahead in time at delayed FI would possibly assist in making conclusions. While we cannot directly attribute differences in outcome to the use of newer anti-fungal agents, these data suggest that these agents may be having an impact on the survivability of FI in the setting of allo-SCT.

Multivariate analyses adjusting for presence of FI, era of anti-fungals, gender, length of stay and insurance status

\begin{tabular}{|c|c|c|}
\hline & OR (95\% CI) & Pr $>$ ChiSq \\
\hline Fungal Infection & $4.331(3.405-5.509)$ & $<0.0001$ \\
\hline Insurance & & \\
\hline Medicare & $1.359(1.066-1.733)$ & 0.0145 \\
\hline Medicaid & $1.187(0.950-1.483)$ & 0.2585 \\
\hline Others & $0.821(0.597-1.128)$ & 0.0273 \\
\hline Private & $1.00($ Ref $)$ & \\
\hline Era of Antifungals & $1.00($ Ref $)$ & \\
\hline New Antifungals (2002 onwards) & $1.830(1.377-2.431$ & $<0.0001$ \\
\hline Before New Antifungals (2000 and 2001) & $3.017(2.428-3.749)$ & $<0.0001$ \\
\hline Length of Stay (LOS) & $1.00($ Ref $)$ & \\
\hline LOS 27 days (Median) & & \\
\hline LOS less than or equal to 27 days (Median) & $1.009(0.859-1.184)$ & 0.9174 \\
\hline Gender & $1.00($ Ref $)$ & \\
\hline Male & & \\
\hline Female &
\end{tabular}

Multivariate analyses adjusting for presence of FI, gender, length of stay and insurance status in 2000-2001 and in 2002-2010

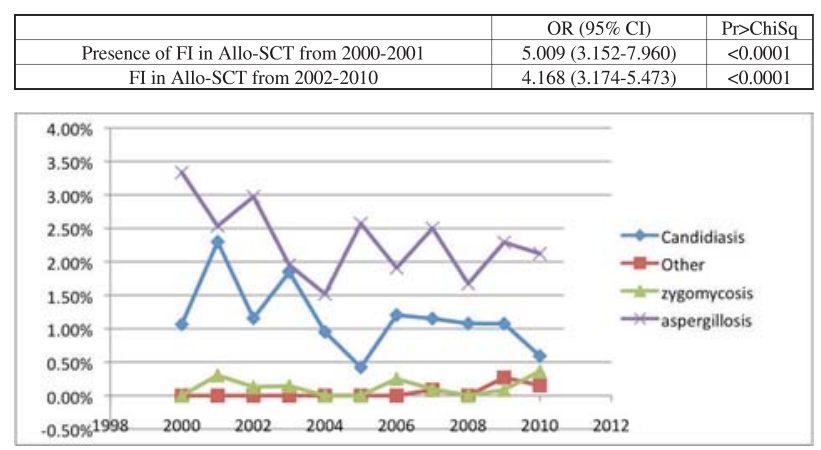

\section{Keywords}

Multiple Myeloma; Stem Cell transplant; Fungal Infection

\section{*Nishi Shah}

E-mail: nishi.shah@emory.edu 